JLO (1984) Limited, 2014

doi:10.1017/S0022215114000516
Nicorandil: ulcer and location

Dear Sirs,

We read with great interest the case reported in this journal by Salim et al. ${ }^{1}$ concerning the ulceration of the nasal dorsum in a 64-year-old man treated with nicorandil (a medical treatment for stable angina). We would like to contribute to the topic confirming a very likely diagnosis of a drug-induced adverse effect, and put forward for consideration two remarks regarding this serious and yet to be sufficiently recognised adverse effect.

The first commentary refers to the conditions of occurrence of ulcers induced by nicorandil, and the second one to their preferential location.

Nicorandil, a nicotinamide ester (N-(2-hydroxyethyl) nicotinamide-nitrate ester), is the first and only association between nitrate and potassium channel activators. Studies on animals have shown that nicorandil is as effective as cimetidine at treating experimentally induced gastric ulceration. In one experiment, Sakai et $a .^{2}$ demonstrated that nicorandil (3 and $10 \mathrm{mg} / \mathrm{kg}$, orally), like cimetidine $(50 \mathrm{mg} / \mathrm{kg}$, orally), protected against acute gastric damage in water immersion plus restraint stress- and aspirininduced gastric ulcer models of rats. However, after intravenous pre-treatment with an antagonist of ATP-sensitive potassium channels (glibenclamide), the ulcer lesions were increased. Ironically, nicorandil was found to be involved in the induction of oral ulcers in 1997 by Reichert et al. ${ }^{3}$ Since then, cases of single or multiple nicorandil-induced ulcerations have been reported, including ulcers at gastrointestinal locations. ${ }^{4}$

According to Trechot et al., ${ }^{5}$ in the particular configuration of high-dosage nicorandil, nicotinamide and nicotinic acid derivatives (two main metabolites of nicorandil) may be unable to merge into the endogenous pool of nicotinamide adenine dinucleotide and nicotinamide adenine dinucleotide phosphate, leading to abnormal in situ distribution of these molecules in the body. Additionally, in the case of recent or maintained trauma, nicotinamide increases blood flow at the edge of the raw area, while nicotinic acid $(\mathrm{pKa}=4.8)$ ulcerates the new epithelial formation. The results of the Sakai et al. ${ }^{2}$ study are not disputed. However, extrapolation of the animal experiments to humans cannot be considered as the animals were killed 5 hours after the administration of a single oral dose of nicorandil. This renders such examination of a delayed adverse effect of nicotinic acid, and study of the metabolite responsible for ulcers, impossible in humans.

Considering that one of the clinical particularities of nicorandil-induced ulcerations is a typical location pattern in areas of trauma, it is necessary to investigate the cause of trauma.
In the case reported by Salim et al., ${ }^{1}$ the use of a full-face mask should be considered as a factor.

\section{P TRECHOT}

Department of Clinical Pharmacology, University Hospital, Nancy, France

\section{References}

1 Salim F, Joshi A, Hopkins C. Ulceration of the nasal dorsum: a rare cause? J Laryngol Otol 2014. Epub 2014 Jan 28

2 Sakai K, Akima M, Katsuyama I. Effects of nicorandil on experimentally induced gastric ulcers in rats: a possible role of K(ATP) channels. Jpn J Pharmacol 1999;79:51-7

3 Reichert S, Antunes A, Trechot P, Barbaud A, Weber M, Schmutz JL. Major aphthous stomatitis induced by nicorandil. Eur $J$ Dermatol 1997;7:132-3

4 Egred M, Andron M, Morrison WL. Nicorandil may be associated with gastrointestinal ulceration. BMJ 2006;332:889

5 Trechot P, Barbaud A, Petitpain N, Claeys A, Schmutz JL. Nicorandil and ulcerations: a NAD/NADP and nicotinic acid-dependent side-effect? Br J Dermatol 2008;158:1150-1

The Journal of Laryngology \& Otology (2014), 128, 386-387. (c) JLO (1984) Limited, 2014

doi:10.1017/S0022215114000528

\section{Authors' reply}

Dear Sirs,

We thank Dr Trechot for his letter and interest in our paper entitled 'Ulceration of the nasal dorsum: a rare cause?' While we appreciate that the location of the ulceration is unusual for a nicorandil-related ulcer, I can reassure Dr Trechot that we considered the use of a full-face continuous positive airway pressure (CPAP) mask as a causative factor of the ulcer. One of the first interventions upon discovering the ulcer was changing the patient's mask to a different low-pressure fitting mask. Furthermore, the patient discontinued using the full-face mask as the ulcer was causing too much pain.

Despite these measures, the ulcer failed to heal after a waiting period of several months. Attempts at primary closure were also unsuccessful. It was not until nicorandil was completely discontinued that the ulcer started to heal. We appreciate that we cannot rule out the full-face CPAP mask as a precipitating factor; however, we feel very confident that nicorandil played a significant role in the ulcer failing to heal in this particular case.

\section{F SALIM}

ENT Department, Guy's Hospital, London, UK 